\title{
Greetings From the New Editor-in-Chief
}

\author{
Ho-Shin Gwak \\ Department of Neurosurgery/Neuro-Oncology Clinic, National Cancer Center, Goyang, Korea
}

Dear colleagues,

It is my great privilege to take over the position of Editor inChief of the "Brain Tumor Research and Treatment (BTRT)," which is the official journal of the Korean Brain Tumor Society (KBTS), the Korean Society for Neuro-Oncology (KSNO), and the Korean Society for Pediatric Neuro-Oncology (KSPNO). These three societies are represented by more than a thousand active members who have joined with enthusiasm for interdisciplinary scholarship and dialogue.

In 2002, this journal began as the Journal of Korean Brain Tumor Society, representing the official journal of the KBTS; however, in 2013, when joined by the KSNO and KSPNO, officially took on its new title as the BTRT. Thanks to the efforts of the first Editor-in-Chief, Professor Heon Yoo (2013-2017) of the National Cancer Center, and the second Editor-in-Chief, Professor Jeong-Hyun Hwang (2017-2021) of Kyungpook National University Hospital, BTRT is regularly published twice a year, and is indexed in PubMed Central, PubMed, KoreaMed, KoreaMed Synapse, and KRF/KCI. Within its publications, the BTRT has encompassed the broad field of central nervous system tumor treatment and research, and proudly represents the birth and growth of the field of neuro-oncology in South Korea.

I begin this appointment with great hope, excitement, and a deep commitment to continue the progress of the BTRT in fulfilling its mission.
I envision the future of the BTRT as follows:

1) Increase publications to quarterly publications: the KBTS, KSNO, and KSPNO hold two scientific meetings annually in addition to seminars. Given this wealth of knowledge contributed by physicians and scientists each year, it is essential that we increase the number of BTRT publications quarterly.

2) Become a journal that embraces physicians and physician-scientists of all levels of experience: we have reinforced our Ethics committee and have invited more editors/reviewers dedicated to scientific exploration who will provide more opportunities to receive excellent peer-review. I believe that this mentorship can be immensely helpful for both junior and senior physicians and physician-scientists in developing or advancing their scientific careers.

3) Become an international journal that represents the neuro-oncology societies of Asian countries: with a spirit of "One Asia" and devotion to those societies unable to have an official journal, BTRT will extend an invitation for membership and help publish their scientific research in BTRT.

Although becoming a SCI(E) journal is not a main goal of $B T R T$, I strongly believe that this can come naturally with dedicated and honorable scientific research and academic pursuit.

In closing, I would like to acknowledge and thank the efforts and devotions of the BTRT editors, reviewers, and editorial office members who have been essential in keeping our journal running.

This is an Open Access article distributed under the terms of the Creative Commons Attribution Non-Commercial License (https://creativecommons.org/licenses/by-nc/4.0) which permits unrestricted non-commercial use, distribution, and reproduction in any medium, provided the original work is properly cited.

Copyright $\odot 2021$ The Korean Brain Tumor Society, The Korean Society for NeuroOncology, and The Korean Society for Pediatric Neuro-Oncology 